\title{
Correction to: Exploring Social Biomarkers in High-Functioning Adults with Autism and Asperger's Versus Healthy Controls: A Cross-Sectional Analysis
}

\author{
Marta Del Valle Rubido ${ }^{1}$ - Eric Hollander ${ }^{2}$. James T. McCracken ${ }^{3}$. Frederick Shic ${ }^{4,5}$. Jana Noeldeke Jan $^{1,7}$ Lauren Boak $^{6}$. \\ Omar Khwaja ${ }^{1} \cdot$ Shamil Sadikhov $^{6} \cdot$ Paulo Fontoura $^{6} \cdot$ Daniel Umbricht $^{1}$
}

Published online: 4 May 2020

(c) The Author(s) 2020

\section{Correction to: \\ Journal of Autism and Developmental Disorders https://doi.org/10.1007/s10803-020-04493-5}

The original version of this article unfortunately contained a mistake in CI values in Table 2.

For Looking preference (ratio), the $90 \%$ CI of estimate should be with a minus sign $-0.15,-0.03$.
The original article can be found online at https://doi.org/10.1007/ s10803-020-04493-5.

\section{Marta Del Valle Rubido}

marta.del_valle_rubido@roche.com

1 Roche Innovation Center Basel, Roche Pharmaceutical Research and Early Development, NRD, Basel, Switzerland

2 Psychiatry and Behavioral Sciences, Albert Einstein College of Medicine and Montefiore Medicine, Bronx, NY, USA

3 Psychiatry and Behavioral Sciences, David Geffen School of Medicine at UCLA, Los Angeles, CA, USA

4 Center for Child Health, Behavior and Development, Seattle Children's Research Institute, Seattle, WA, USA

5 Department of Pediatrics, University of Washington, Seattle, WA, USA

6 Roche Product Development Neuroscience, Basel, Switzerland

7 Roche Global Product Strategy Neuroscience, Basel, Switzerland
For Human activity preference (ratio), the $90 \% \mathrm{CI}$ of estimate should be with a minus sign $-0.30,-0.08$.

The correct Table 2 is given below.

The original article has been corrected. 
Table 2 Eye tracking data for ASD and HC groups

\begin{tabular}{|c|c|c|c|c|c|c|}
\hline $\operatorname{Task}^{\mathrm{a}}$ & $\begin{array}{l}\text { Mean ASD } \\
(\mathrm{N}=38)\end{array}$ & $\begin{array}{l}\text { Mean control } \\
(\mathrm{N}=19)\end{array}$ & $\begin{array}{l}\text { Estimate of differ- } \\
\text { ence ASD-HC }\end{array}$ & $90 \%$ CI of estimate & $P$ value & Cohen's $\mathrm{f}^{2}$ \\
\hline \multicolumn{7}{|l|}{ Activity monitoring } \\
\hline Activity (ratio) & 0.36 & 0.31 & 0.05 & $-0.01,0.11$ & 0.19 & 0.34 \\
\hline Background (ratio) & 0.26 & 0.22 & 0.04 & $-0.010,0.08$ & 0.20 & 0.13 \\
\hline Body (ratio) & 0.13 & 0.11 & 0.03 & $-0.0003,0.05$ & 0.11 & 0.16 \\
\hline Distractors (ratio) & 0.13 & 0.10 & 0.03 & $-0.006,0.06$ & 0.17 & 0.16 \\
\hline Head (ratio) & 0.25 & 0.37 & -0.11 & $-0.16,-0.07$ & $0.00019^{\mathrm{b}}$ & 0.65 \\
\hline Person (ratio) & 0.38 & 0.47 & -0.09 & $-0.14,-0.04$ & 0.005 & 0.52 \\
\hline \multicolumn{7}{|l|}{ Biodetection } \\
\hline$d$-prime (masked condition) & 1.53 & 1.58 & -0.06 & $-0.65,0.54$ & 0.88 & 0.21 \\
\hline Latency (ms) & 300.37 & 274.34 & 26.02 & $-20.23,72.28$ & 0.36 & 0.11 \\
\hline Looking preference (ratio) & 0.60 & 0.69 & -0.09 & $-0.15,-0.03$ & 0.02 & 0.35 \\
\hline Orienting preference (ratio) & 0.49 & 0.50 & -0.01 & $-0.05,0.03$ & 0.62 & 0.03 \\
\hline \multicolumn{7}{|l|}{$W A V W$} \\
\hline Background (ratio) & 0.13 & 0.12 & 0.01 & $-0.01,0.04$ & 0.39 & 0.20 \\
\hline Body (ratio) & 0.10 & 0.08 & 0.02 & $-0.01,0.05$ & 0.28 & 0.21 \\
\hline Eyes (ratio) & 0.40 & 0.42 & -0.03 & $-0.11,0.06$ & 0.59 & 0.10 \\
\hline Head (ratio) & 0.74 & 0.78 & -0.03 & $-0.09,0.02$ & 0.27 & 0.22 \\
\hline Mouth (ratio) & 0.25 & 0.26 & -0.01 & $-0.09,0.06$ & 0.76 & 0.09 \\
\hline \multicolumn{7}{|l|}{ Gaze discrimination } \\
\hline Eyes (ratio) & 0.38 & 0.37 & 0.01 & $-0.07,0.09$ & 0.84 & 0.04 \\
\hline Inside face (ratio) & 0.92 & 0.94 & -0.02 & $-0.07,0.02$ & 0.38 & 0.049 \\
\hline Mouth (ratio) & 0.06 & 0.06 & 0.008 & $-0.03,0.05$ & 0.71 & 0.01 \\
\hline Nose (ratio) & 0.33 & 0.38 & -0.05 & $-0.12,0.01$ & 0.19 & 0.13 \\
\hline \multicolumn{7}{|l|}{ Gender discrimination } \\
\hline Eyes (ratio) & 0.22 & 0.18 & 0.04 & $-0.02,0.09$ & 0.33 & 0.14 \\
\hline Inside face (ratio) & 0.91 & 0.93 & -0.03 & $-0.08,0.03$ & 0.39 & 0.05 \\
\hline Mouth (ratio) & 0.09 & 0.07 & 0.02 & $-0.02,0.07$ & 0.42 & 0.06 \\
\hline Nose (ratio) & 0.45 & 0.54 & -0.09 & $-0.16,-0.01$ & 0.06 & 0.20 \\
\hline \multicolumn{7}{|c|}{ Human activity preference (Social vs Geometric) } \\
\hline Human activity preference (ratio) & 0.60 & 0.79 & -0.19 & $-0.30,-0.08$ & 0.007 & 0.32 \\
\hline \multicolumn{7}{|l|}{ Total } \\
\hline Composite score & -0.08 & 0.41 & -0.48 & $-0.86,-0.11$ & 0.04 & 0.30 \\
\hline
\end{tabular}

Estimate refers to estimated mean differences between ASD and HC derived from an analysis of Covariance model

$P$ values less than 0.00094 are considered statistically significant after multiplicity adjustment

${ }^{a}$ Categories in each task (e.g. Activity, Background) refers to the time spent looking at this aspect of the visual scene in relation to the overall looking time

${ }^{\mathrm{b}}$ Measure that survived Bonferroni correction

$A S D$ Autism spectrum disorder, $H C$ healthy control, WAVW Who's afraid of Virginia Woolf

Publisher's Note Springer Nature remains neutral with regard to

jurisdictional claims in published maps and institutional affiliations. 